embedding, large lexicons, or the lack of iconicity, which seem to serve communication purposes at the expense of learnability.

Christiansen \& Chater $(\mathrm{C} \& \mathrm{C})$ rightfully observe that communicatively arbitrary principles, such as UG, are unable to explain why language is adequate for communication. The same criticism can be addressed, however, to their own account. If the main driving force that led to language emergence is learnability rather than communicative efficiency, language should be locally optimal for the former and not for the latter. Evidence suggests that, in several respects, the exact opposite is the case.

What would language be like if, as $\mathrm{C} \& \mathrm{C}$ claim, the cultural selection of learnable languages were "stronger" than the biological selection of brains designed for efficient communication? If language can compare with a "viral" entity that gets selected for its ability to resist vertical cultural transmission, we predict, for instance, iconic signifiers, especially gestures, to win the contest. Yet, although analogue resemblance makes learning almost trivial, linguistic evolution shows that non-iconic signifiers tend to prevail, even in sign languages.

The "viral" theory of language does not explain the size of lexicons either. Ideally, an expressive code is easiest to learn, and resists iterated transmission best, if words are limited in number and have separate and unambiguous meanings. Yet, real vocabularies include tens of thousands of words, massive near synonymy, and many rare unpredictable word combinations (Briscoe 2006). Such evidence suggests that there may be some "viral" cause for the existence of plethoric lexicons, but its action is opposite to what is expected from selection for learning efficiency.

Language, as mainly shaped by selection through repeated learning, is supposed to mirror the general human induction bias. Efficient induction systems (Solomonoff 1978), including human learning (Chater 1999) and analogy making (Cornuéjols 1996), are guided by a complexity minimization principle. If languages were the bare expression of a simplicity-based induction device looping on itself, we should expect the complexity of languages to converge to a minimal amount. A similar claim is that general-purpose learning devices, except in rote learning mode, produce only "good shapes" (Gestalten) - that is, structures that are left invariant by operations forming an algebraic group (Dessalles 1998a). Language has not, so far, been described as involving good shapes. For instance, syntactic structures, contrary to many other aspects of cognition, cannot be induced as invariants of transformation groups (Piattelli-Palmarini 1979) and seem to thwart general inductive processes (Piattelli-Palmarini 1989).

In a bio-functional account of language emergence, learnability puts limits on what is admissible, but is subordinate to communicative functions. The two main proximal functions of language in our species, as revealed by the observation of spontaneous language behavior, are conversational narratives and argumentative discussion (Bruner 1986; Dessalles 2007). From a bio-functional perspective, iconicity is dispensable if the problem is to express predicates for argumentative purposes (Dessalles 2007). Lexical proliferation is predicted if the problem is to signal unexpectedness in narratives and to express nuances in argumentative discussion (Dessalles 2007). And language-specific learning bias is expected if early language performance makes a biological difference. Let us consider a fourth example to show that functional aspects of language could evolve at the expense of learnability.

Non-functional accounts of language, including cultural selection through iterated learning, do not account for the existence of central embedding (the fact that any branch may grow in a syntactic tree), a feature present in virtually all languages. Recursive syntax has been shown to emerge through iterated learning, but only when individuals already have the built-in ability to use recursive grammars to parse linguistic input (e.g., Kirby 2002). A bio-functional approach to language provides an explanation for the presence of central embedding in language. As soon as the cognitive ability to form predicates is available, possibly for argumentative purposes (Dessalles 2007), predicates can be recruited to determine the arguments of other predicates. This technique is implemented in computer languages such as Prolog. To express "Mary hit Paul" for listeners who do not know Mary, the speaker may use "Mary ate with us yesterday" to determine the first argument of "hit." Prolog achieves this through explicit variable sharing, whereas human languages connect phrases for the same purpose: "The girl who ate with us yesterday hit Paul” (Dessalles 2007).

Predicates $P_{1 i}$ can therefore be used to determine arguments in a given predicate $P_{1}$; but each $P_{1 i}$ may require further predicates $P_{1 i j}$ to determine its own arguments. This possibility leads to recursive syntactic processing that produces central embedded phrase structures. Models that ignore functions such as predicate argument determination cannot account for the necessity of embedded phrase processing. They merely postulate it, either as a consequence of some fortuitous genetic accident (Chomsky 1975 ) or deduced from a general cognitive ability to perform recursive parsing (Kirby 2002). But then, the adequacy to the function is left unexplained as well. No single genetic accident and no selection through repeated learning can predict that phrase embedding will efficiently fulfill predicate argument determination. Only a bio-functional approach that derives the existence of phrase embedding from its function can hope to explain why recursive processing came to exist and why it is locally optimal for that function.

From a phylogenetic perspective, we may wonder why, if human languages have been selected to be easily learned, chimpanzees are so far from acquiring them, spontaneously or not. One must hypothesize some yet unknown qualitative gap between animal and human general learning abilities. Invoking such "pre-adaptation" remains, for now, non-parsimonious. Not only is the emergence of "pre-adaptations" not accounted for in iterated learning models and more broadly in non-functional models, but their subsequent assemblage into a functional whole remains mysterious as well. Bio-functional approaches to language emergence avoid the "pre-adaptation" trap. They do not attempt to explain why a given feature did not occur in other lineages by invoking the lack of required "pre-adaptations."

Language is not a marginal habit that would be incidentally used in our species. It has dramatic influence, not merely on survival, but on differential reproduction, which is what determines natural selection. Individuals who fail to be relevant are excluded from social networks and become preferential victims (Dessalles 1998b; 2007). Given the crucial impact of conversational performance on reproductive success, it would be highly unlikely that human brains could have evolved independently from language.

\section{Language as shaped by social interaction}

doi:10.1017/S0140525X08005104

\section{N. J. Enfield \\ Language and Cognition Group, Max Planck Institute for Psycholinguistics, 6500 AH, Nijmegen, The Netherlands. \\ Nick.Enfield@mpi.nl http://www.mpi.nl/Members/NickEnfield}

Abstract: Language is shaped by its environment, which includes not only the brain, but also the public context in which speech acts are effected. To fully account for why language has the shape it has, we need to examine the constraints imposed by language use as a sequentially organized joint activity, and as the very conduit for linguistic diffusion and change. 
I welcome Christiansen \& Chater's (C\&C's) contention that cognitive scientific approaches to language should take seriously the idea that language is adapted to its environment. Although C\&C concentrate on the private cognitive and neural structures that each language user possesses, my commentary concerns a different aspect of the environment of language, one which is no less responsible for the shape language takes: namely, the public social-interactional setting in which language is learned and used, and which is the central conduit for the historical distribution and development of language in populations.

$\mathrm{C} \& \mathrm{C}$ consider the implications of a dual-inheritance model by which human genetic evolution proceeds in parallel with much faster processes of historical evolution of culture in domains such as kinship, social values, technology, and language (Cavalli-Sforza \& Feldman 1981; Durham 1991; Richerson \& Boyd 2005; cf. Enfield 2005; 2008, for language). A great discrepancy between the high speed of language change and the relatively low speed of genetic evolution drives C\&C's argument that language (evolving historically) is adapted to the brain (evolving phylogenetically), rather than the other way around. This requires that language change be analyzed in evolutionary terms. To this end, C\&C draw a parallel between the language system and the organism, as others before them have done. However, this may not be the most apt analogy. In genetic evolution, the organism serves as a vehicle for the replication of genes. In language, the vehicle for replication of linguistic units (e.g., words or constructions; Nettle 1999) is not the language system as a whole. The larger system is an aggregate of interrelated linguistic items. It may be stored in individual brains, or in linguistic descriptions, but it is not a vehicle for replication of linguistic units.

The vehicle by means of which linguistic items are used and diffused is the speech act (or utterance; Croft 2000). Through being used in speech acts, linguistic items maintain circulation in populations; and it is through this circulation that selection of linguistic variants takes place. Diffusion of linguistic variants involves not just brains, but a see-sawing process, from private mental states (conceptual representations, communicative intentions), to public states of affairs (speech acts), back to new brain states (interpretations of speech acts leading to new conceptual representations, new communicative intentions), and on to new states of affairs (more speech acts). It is a continual chain of transition from private to public to private to public, and so on (Sperber 2006) - as is the case in the historical evolution of cultural variants more generally (Richerson \& Boyd 2005; Rogers 1995).

Linguists of many stripes recognize the privileged status of something closely akin to the speech act as the basic shape for linguistic organization: variously described, for example, as the "clause" (Foley \& Van Valin 1984), "intonation unit" (Chafe 1994), "turn-constructional unit" (Sacks et al. 1974), "growth point" (McNeill 1992), and so on. Why should just this unit constitute the privileged shape for linguistic organization? The answer is that the speech act or utterance is a basic unit at the level of informational delivery; that is, an utterance conveys one idea at a time (Pawley \& Syder 2000), thereby effecting one increment of social action at a time (Heritage \& Atkinson 1984). Is there something about the brain that privileges linguistic units of just this shape? Or are there other reasons that the speech act (utterance, clause, turn, etc.) should be a privileged shape for linguistic structure?

There is reason to think that the one-speech-act-at-a-time structure of grammar is shaped by contingencies of the conversational settings in which language is learned, used, and diffused. Human social interactions are sequences of moves and counter-moves (Goffman 1964), each move being a response to an immediately prior move, and a stimulus for a subsequent move (Sacks et al. 1974). This pattern of rapid response and counter-response is what characterizes free conversation, the basic format for language acquisition and use in everyday life. ${ }^{1}$ Once we view the use of language in human interaction as a type of joint activity (Clark 1996b), and not just an event of information processing that happens to involve more than one brain (Hutchins 1995; 2006), then we see how language is shaped by the sequential contingencies of social interaction. Grammar's basic "chunking" delivers minimal units, such that each unit contributes to a sequence of social interaction as an adequate response to a prior unit (Schegloff 2006). To be effective, any such unit increment must not only be successfully formulated by a speaker, but must also be successfully comprehended by a listener. The organization of language in chunks of "turn" size gives interlocutors the opportunity to monitor misfirings and misunderstandings as soon as they happen, and to correct them if necessary (Schegloff et al. 1977). This co-contingency of unit contribution and response may be argued to serve as a direct determinant shaping linguistic organization, including many of the properties of "Universal Grammar" that C\&C are looking to explain without reference to an innate language faculty (cf. Tomasello 1995; 2004).

In sum, the acquisition and use of language involves not just the brain's private structures, but also the public patterns of codependent, interlocking contributions to sequences of social interaction. The preferred locus of grammatical organization the speech act - is an optimal solution to multiple constraints of information-packaging in an environment where not just formulation of social action, but the possibility of timely monitoring of intersubjective understanding and appropriateness of response is as definitive of linguistic well-formedness as any arbitrary unit of sentence-level syntactic structure.

\section{NOTE}

1. Surprisingly little is known about the structure of language in conversation, apart from a rich body of work on English (see Schegloff [2007] and references therein). Comparative work on conversational structures is beginning to appear (see Enfield \& Stivers 2007; Sidnell 2007).

\section{The origin of language as a product of the evolution of double-scope blending}

\section{doi:10.1017/S0140525X08005116}

\section{Gilles Fauconnier ${ }^{\mathrm{a}}$ and Mark Turner ${ }^{\mathrm{b}}$ \\ ${ }^{a}$ Department of Cognitive Science, University of California, San Diego, La Jolla, CA 92093; ${ }^{\mathrm{b}}$ Department of Cognitive Science, Case Western University, Cleveland, $\mathrm{OH} 44106$. \\ faucon@cogsci.ucsd.edu \\ http://www.cogsci.ucsd.edu/ faucon/ turner@case.edu http://markturner.org}

Abstract: Meaning construction through language requires advanced mental operations also necessary for other higher-order, specifically human behaviors. Biological evolution slowly improved conceptual mapping capacities until human beings reached the level of doublescope blending, perhaps 50 to 80 thousand years ago, at which point language, along with other higher-order human behaviors, became possible. Languages are optimized to be driven by the principles and powers of double-scope blending.

Christiansen \& Chater $(\mathrm{C} \& \mathrm{C})$ are correct in their claim that it is implausible that language as we know it is the product of biological evolution for a complex, language-specific endowment. As they point out, this is not in itself an unusual claim. For example, Hauser et al. (2002) argue that the sole language-specific capacity might be recursion, and that even recursion might not be language-specific. However, our reasoning to this claim is quite different from C\&C's. Language as we know it requires advanced capacities for meaning construction. Any view of language as having been active in quasi-advanced forms before about 50 thousand years ago, advancing further by refinements, runs up against the unlikelihood that human beings who enjoyed these nearmodern capacities of meaning construction through language 